\title{
GENETICS OF SHELL GROUND COLOUR IN HELIX ASPERSA I. COLOUR LOCUS, UNIFORM AND THEIR INTERACTIONS
}

\author{
R. M. ALBUQUERQUE DE MATOS \\ Centre of Genetics and Molecular Biology, Ave. Prof. Gama Pinto 2, \\ 1699 Lisbon Codex, Portugal
}

Received 4.xi.83

\begin{abstract}
SUMMARY
In Helix aspersa the shell may present basically three colours, yellow, red and brown, each due to an allele of locus $C$. Heterozygotes as a rule are of intermediate colour, but in some lines one allele may more or less dominate. Colour is better scored in young, adults acquiring a brownish tinge due to periostracum tanning. Another colour locus, not linked with $C$, is $U$, dominant, for uniformly distributed reddish dark brown colour epistatic over the effects of all $C$ alleles. $U$ also suppresses all types of band, the manifestation of darker upper part of the shell and the development of fascia albata, producing a shell surface devoid of structural markings. Effect of $U$ on bands is distinct from that due to allele $M^{0}$ of the locus for intensity of band pigmentation, which does not affect shell structure and colour. Separation of the several effects of $U$ by crossing-over has not been detected. One observed mutation from the recessive $u$ to $U$, which at once produced all the effects of $U$, is in favour of these being truly pleiotropic, not due to closely linked genes.
\end{abstract}

\section{INTRODUCTION}

Genetic determination of shell pigmentary characters in Helix aspersa and similar snails relates essentially to the shell ground colour and the banding systems. The latter is in aspersa very complex and will be considered here only in so far as reference to banding is necessary to understand interactions between bands and shell ground colour. Adult aspersa snails do not in general show bright shell colours as for example those observed in Cepaea nemoralis. However, bright yellows and reds, besides the more usual browns, occur in aspersa in several locations in Portugal, especially in young individuals. In previous genetic work on this species by other authors (for reviews including other Pulmonata or Mollusca cf. Murray, 1975; Clarke et al., 1978) reference has been made to pale and dark browns and to reddish-browns (Cook, 1969) and to yellowish-browns and reddish-browns (Cain, 1971). From extensive observations and breeding work which now has lasted for seven years we conclude, as the evidence discussed in this paper shows, that there are in aspersa three basic shell colours, yellow, red and brown. Each of these basic colours occurs in several hues and shades, so that some homozygous reds appear to approach yellows on one side of their range of variation and browns on the other side, and the same happens relative to the other two colours with some homozygous yellows and browns. Heterozygotes between two of the colours are as a rule intermediate.

Colour is in aspersa due to both the periostracum and the pigment that tinges the calcareous part of the shell. In young individuals the first of these 
components dominates, whereas in adults the two effects combine, but the periostracum tends to become tanned. Hence the usual brownish tinge, which nevertheless does not preclude the basic shell colour from being observed in adult shells, especially on their underside. It may be noted that frequently the upper shell half is darker than the lower half due to the effect of a dominant factor, $S$, that further obscures shell colour (Albuquerque de Matos, 1979).

The main loci here considered are that for the three basic colours and another, Uniform, which interacts with it. It is shown that these two loci are not linked. Evidence in favour of the effects of Uniform on colour being pleiotropic to other effects of the locus is briefly discussed. Notation employed follows the recommendations of the 2 nd International Conference on Snail Genetics, York, England, 1967, in the case of loci where there is no clear dominance of one allele over others. In general these loci show multiple allelism. Their base symbol is capitalised. Loci with clearly two alternative phenotypes, one of which corresponds to the dominant constitution and the other to the recessive, are denoted as usual in genetics, a small letter base for the recessive alleles and the corresponding capitalised base for the dominant alleles. In this way the important item of information constituted by the dominant-recessive relationship is conveyed by the notation, as is the case, for example, in denoting in this paper by $u$ the recessive non-uniform or in denoting by $a$ the recessive allele for albino, a notation used in general in species where albinos are known.

\section{LINES AND CULTURE METHODS}

Lines are derived from young snails or broods of adults obtained from natural populations and designated by the initial letter of the locality of origin (in this paper $C$ for Coimbra, $F$ for Figueira da Foz and $L$ for Lisbon) or the relevant characteristic ( $A$ for albino lines), followed by a Roman figure indicating the order of establishment.

Details of culture methods (laboratory cultures in controlled conditions) have been described in Albuquerque de Matos (1982). Breeding pairs, put together before they become adult, are kept in glass jars of suitable size with a convenient layer of soil covered with dried leaves. If a previously fertilised adult snail is mated to a virgin snail, only the broods laid by this latter are considered in the results and this is expressly stated. As soon as possible after laying, the clutches of eggs are isolated from the breeding jars in plastic boxes and the young are transferred to larger boxes at suitable intervals at which the phenotypes are scored. Food consists of vegetables, mainly lettuce, turnip greens and carrots. Flour enriched with milk solids and powdered calcium carbonate is always provided.

Shells are prepared carefully, avoiding alteration of colours by alkalis or strong detergents and those of significant matings are kept in collection.

\section{Results ON THE $C$ LOCUS}

In populations of the Lisbon region, with which we mainly began our work, yellows are much less frequent than browns and heterozygotes may be difficult to distinguish or indistinguishable from light browns. In such cases observation during shell growth, supplemented by observation of the 
prepared, dried shells may be needed to score yellows with certainty as distinct from light browns and both from their heterozygotes. The first matings we studied (Albuquerque de Matos, 1979) involved browns only or browns and heterozygous yellow-browns from two Lisbon lines where colour scoring was affected by these difficulties. In subsequent work the scoring became surer owing to repeated observation during growth of the snails, followed, if need be, by observation of the prepared dried shells. In populations from other Portuguese locations in the Central and Northerly regions, for example Alcobaça, Coimbra, Figueira da Foz, Braga, etc., the distinction between yellows, browns and their heterozygotes is in general rather easy, but in case of doubt the two procedures just mentioned may be employed. Reds appear also in the populations of every location we have studied, usually as heterozygotes with brown. To them applies what is valid for distinguishing between the other two colours, but it may be noted that in dried shells the red pigment fades more rapidly than the others.

In table 1 results of a few series of matings are indicated, of the few hundreds with certainly scored shell ground colour that, as a part of our breeding work, are informative on this character. In the first two series of matings, 93 through 543A, and 96 through 276, browns, yellow-browns and yellows were mated or segregated. Brown and yellow proved to be homozygous and yellow-brown heterozygous. Numbers obtained agree well with expectation for two alleles of a colour locus $\left(\chi^{2}\right.$ of the segregations with probabilities $\mathrm{P}$, in order, $0.68,0.46,0.54,0.42,0.65,0.63)$. Red was introduced into the next series of matings, 64 through 291, in which four or two classes segregated, as is indicated, showing that red is the third allele of the colour locus ( $\mathrm{P}$ of segregations, in order, $0.32,0.67,0.38$ ). In every case the colour classes could be easily scored, the lines being from the NorthCentral part of the Country. Heterozygotes begin their development with the lightest colour determined by their genotype, i.e. with yellow or red, according to the case, and then gradually the other pigment appears and mixes with the light one. This pigmentary succession remains observable along all the spire or on the underside of the shell till the tanning of the periostracum which frequently takes place in aged adults occur.

The three alleles of the $C$ locus for ground shell colour may be denoted $C^{A}, C^{V}$ and $C^{C}$, for yellow, red and brown respectively (superscripts from the Portuguese words for "amarelo", yellow, "vermelho", red and "castanho", brown, as proposed in our previous papers). Each of the alleles probably has some variants that differ from one another as to hue and/or shade, but this point remains to be studied. In the brown alleles, which we have more extensively studied as to these differences, effects tend to be additively intermediate, or some predominance of lighter alleles may be noted (Albuquerque de Matos, 1979).

The last series of matings indicated in table 1 , from the initial 18 through 416 , refers to the relations between ground colour and intensity of band pigmentation, a point here mentioned because the next locus to be discussed is a band suppressor. There are in $H$. aspersa genes which affect the number and type of bands, as also the fusion of these, but the basic factors, whose expression is indispensable so that other banding factors may also be expressed, are those for intensity of band pigmentation or melanization. Results on this character may be explained (Albuquerque de Matos, 1979) by postulating five alleles of a locus $M$ whose lower allele $M^{0}$, for absence 


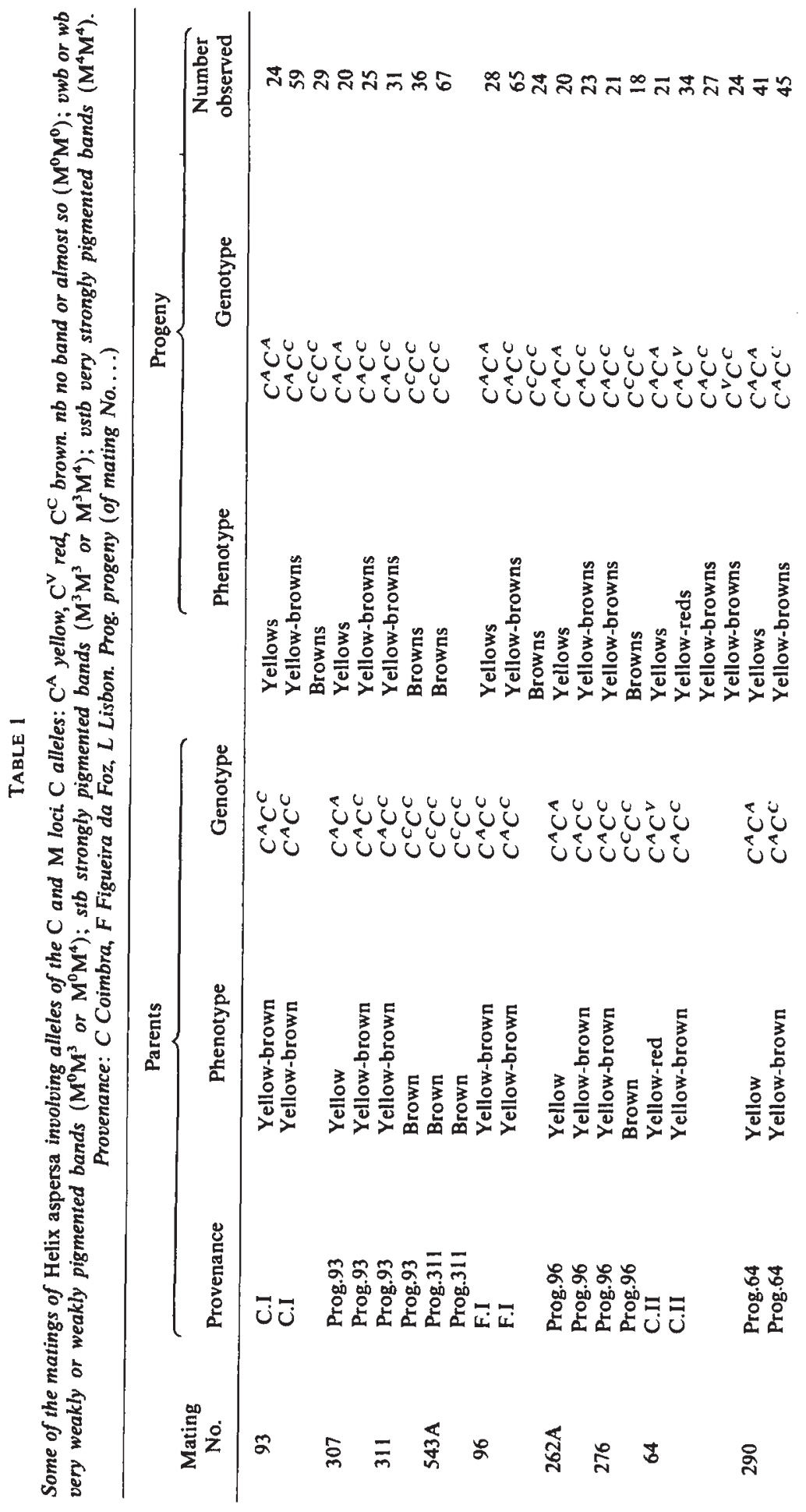




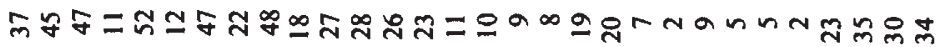

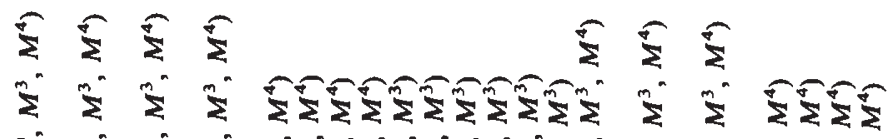

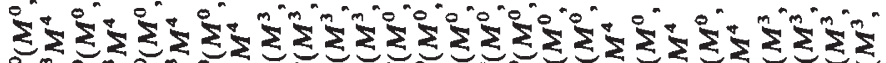

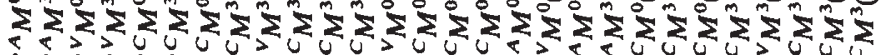

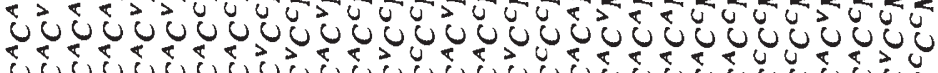

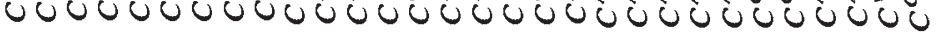

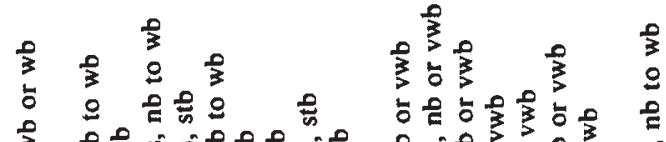

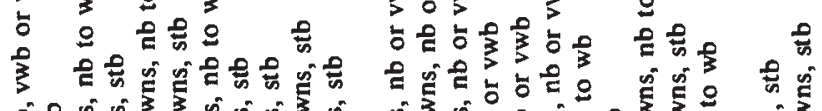

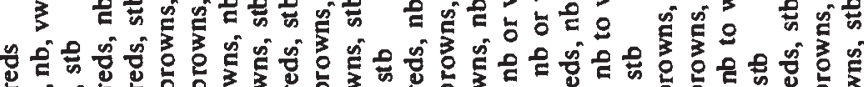

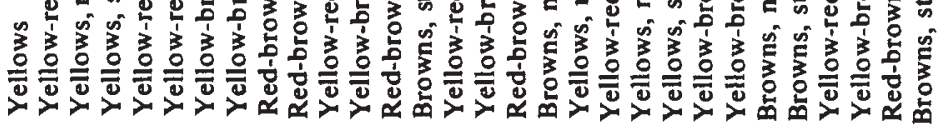

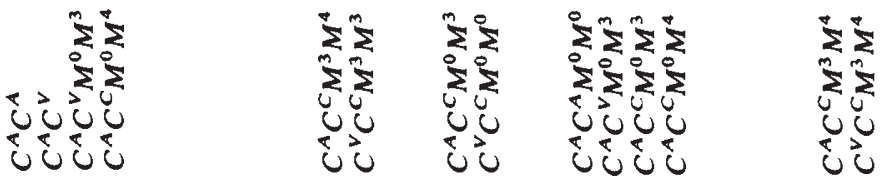

\begin{tabular}{|c|c|c|c|}
\hline 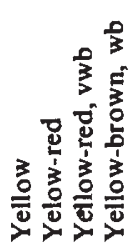 & 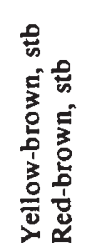 & 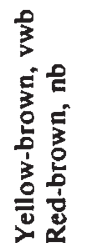 & 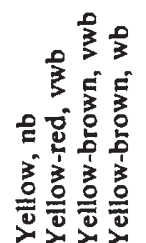 \\
\hline
\end{tabular}

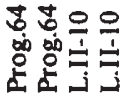

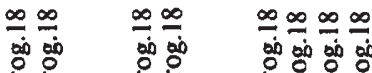

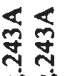

0

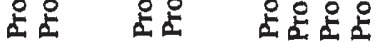

है.

$\overline{2} \infty$

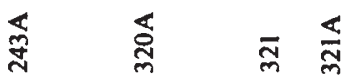

$\frac{0}{7}$ 
of bands or those very weakly pigmented, is dominant or almost so over all the others. The second allele, $M^{l}$, is still rather dominant, whilst the other three, $M^{2}$ to $M^{4}$, for successively increasing intensity of band melanization, produce additively intermediate heterozygotes. To every genetic constitution a somewhat variable expressivity corresponds, less so, however, in the extremes, with $M^{0}$, or with $M^{3}$ and $M^{4}$ alone. The variability does not preclude a reasonable correspondence between genotype and phenotype, especially if middle alleles $\left(M^{2}\right.$ and, although not so necessarily, $\left.M^{1}\right)$ are excluded from the genetic constitution of the mates. This is the case of the series beginning with mating 18 . An L.II strain was chosen where ground colour is scorable with certainty. The results and their interpretation in terms of two distinct and not linked loci are indicated. Three alleles of the $C$ locus and also three of the $M$ locus, the latter postulated so as to correspond to the scored intensities of band pigmentation, are involved in the segregations, with unequivocal results $(\mathrm{P}$, in order, $0.80,0.91,0.91$, $0.87,0.82$ and 0.40 ).

\section{RESULTS ON THE $U$ LOCUS AND $U-C$ INTERACTION}

The uniform strain here considered originated from snails of a single brood of a mixed Lisbon IV population reared in the laboratory. Uniform L.IV snails show a reddish dark brown shell of uniform colouration, neither with bands nor with the usual differences in pigmentation between the upper and lower shell halves, and also the shells show a uniformly fine wrinkled surface, without structural markings. Similar snails as to shell surface and lack of bands have also appeared in two other Lisbon lines and are being studied. Other unbanded snails, which occur not infrequently in aspersa populations, are due to the presence in their genotype of the $M^{0}$ allele above alluded to. Absence or strong reduction of bands is not in this case associated with any particular ground coloration and shell structure is not markedly changed relative to that of banded shells.

The unbanded condition of the uniform L.IV strain has proved to be due to a dominant factor $U$, a suppressor of all types of band and also of shell structural markings or mottling (Albuquerque de Matos, 1979). The reddish dark brown colour associated with the other two effects of $U$, on bands and on shell structure, has also been shown to be dominant or epistatic over the shell colours due to the $C$ locus (Albuquerque de Matos, 1982). Here this point is dealt with on the basis of more complete data and new facts on the nature of the relation between effects of $U$ on pigmentation and on bands and shell texture as also new studies of the interactions between $U$ and $C$, are reported. New data prove conclusively that $U$ and $C$ are not linked.

Table 2 shows two of the several series of matings which were studied, respecting to segregations of $U$ and $C$ and to interactions of these loci. In the first series, matings 52 through 575 , the mates in the initial cross were both from line L.IV, one uniform and the other banded of yellow-brown colour. Four classes were scored in the progeny, one like the uniform parent as to colour and shell structure, the other three banded and of three colours, with numbers in each class as indicated. Five sib crosses from this progeny followed: one between uniforms (229), two between a uniform and a banded (229A, 232), and two between banded sibs $(233,235)$. All segregated in 
agreement with expectation for the genotype indicated. The genotypes of uniforms as to $C$ alleles present were inferred from the colour classes which segregated in their banded progenies. Direct scoring of the uniforms for colour due to $C$ alleles present in their genotype is possible, as will be shown in the following paper of this series, when one albino allele weakens the epistatic effect of $U$ on the manifestation of $C$.

The second series of matings listed in table 2 begins with mating 325 , between an L.IV uniform and an F.I banded yellow-red. Four other matings follow. This series includes the red $C^{v}$ allele not present in the first series and is of interest because it includes also a uniform snail (mating 510A) from whose genotype the brown allele $C^{C}$ surely was absent. Results agree with those of the first series of matings, but the fact that the reddish dark brown shell colour of uniform shells develops without the presence of a $C^{C}$ brown allele in the genotype indicates that such a colour is not due to some effect of $U$ on this allele. Progeny numbers agree well in every mating of the two series with expectation for epistasis of $U$ over colour effects of $C$ alleles which are present in the genotype, the two loci segregating independently ( $P$ from 0.40 to 0.79 for progenies of matings following 52; and $P$ from 0.52 to 0.86 for progenies of matings following 325 ).

\section{(a) Effects of $U$ and interactions with $C$}

It is somewhat unexpected that a single locus has three types of effect, on bands, shell structure and shell ground colouration. In the several tens of matings involving $U$, with a few thousands of offspring in which the separation by crossing-over of these three effects could be detected, we have never observed such a separation. It might be thought that the effect on shell structure would simply be due to the suppression of the bands, but the lack of these latter caused by $M^{0}$, above alluded to, is not accompanied by suppression of structural markings as in uniform. $\boldsymbol{M}^{0}$ may produce some attenuation of the mottling usually seen in banded aspersa, but the effect of $U$ in this respect is much more accentuated. $M^{0}$ also has no effects on darker upper part of the shell due to the dominant $S$ and on fascia albata, due to the dominant $F a$.

The effect on ground colour might be supposed to be due to an allele of the same locus $C$ that determines the other colours but, as was shown above, $U$ and $C$ not only are distinct loci, but also they are not linked. The colour produced by $U$ is similar to that of some dark brown alleles $C^{C}$, but the uniform colouration includes a reddish hue which distinguishes it from all colours due to $C$. Perhaps the colour effect of $U$ could be due to enhancement by this locus of the effect of $C$ when this determines a brown coloration, or in another way $U$ might include part of the $C$ effects, a brown allele of this locus having been translocated to near the gene segments that in $U$ produce the effects on bands and shell structure. The first of these possibilities is disproved by the fact that, as shown in table 2, presence in the genotype of a brown allele of $C$ is not needed for the colour effect of $U$ to manifest itself. As to the second possibility, we have never detected any anomaly in segregations involving $U$ and $C$ which might be expected to occur if a rearrangement involving these two loci was present in the genotypes of the lines employed in the matings

Effects of $U$ on shell structure may be more dominant than the other effects. $U u$ heterozygotes may show band vestiges, especially as pigmented 


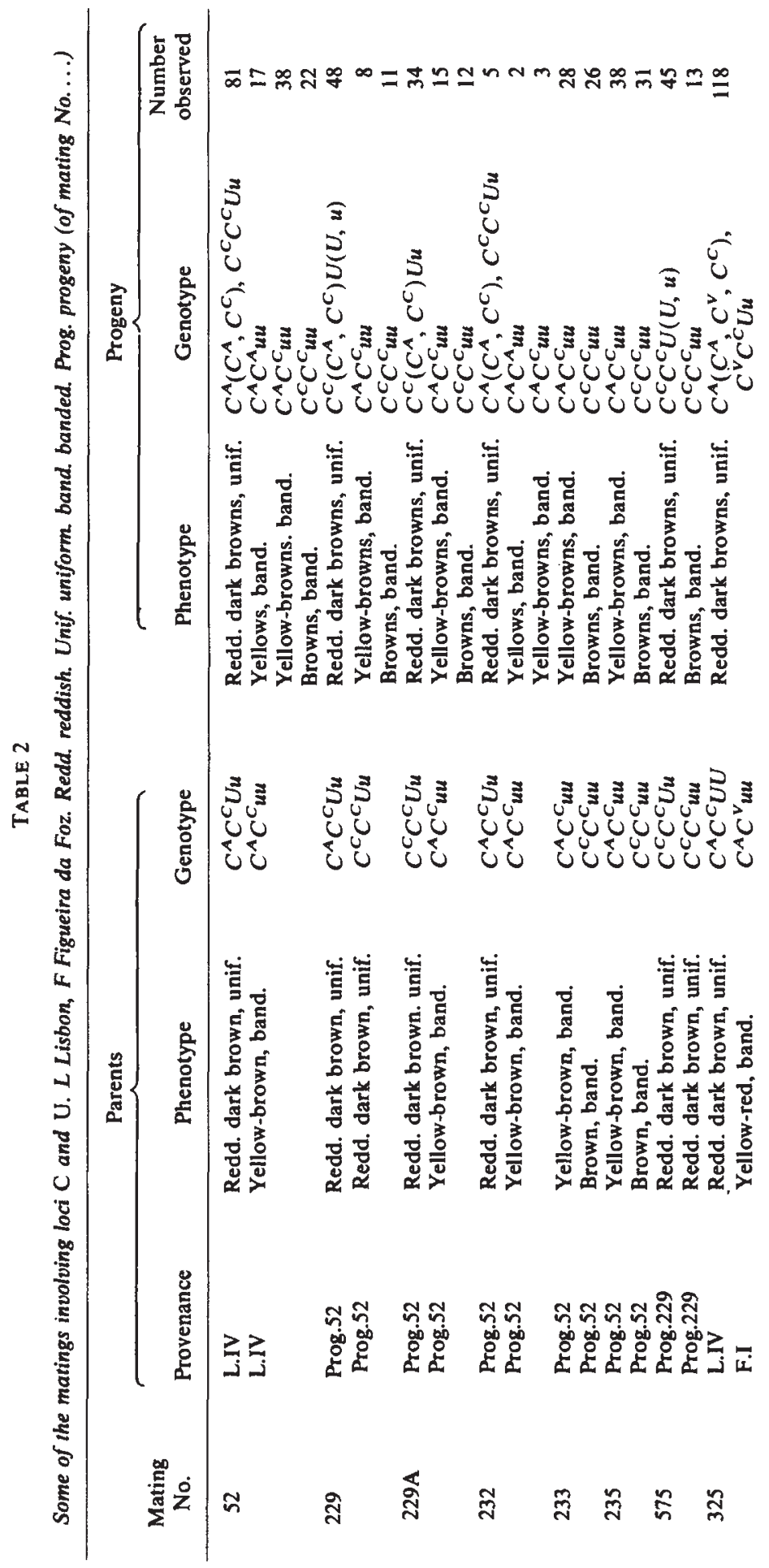


요

$\rightarrow n+n=-m m-\infty$ mnmog -nm-
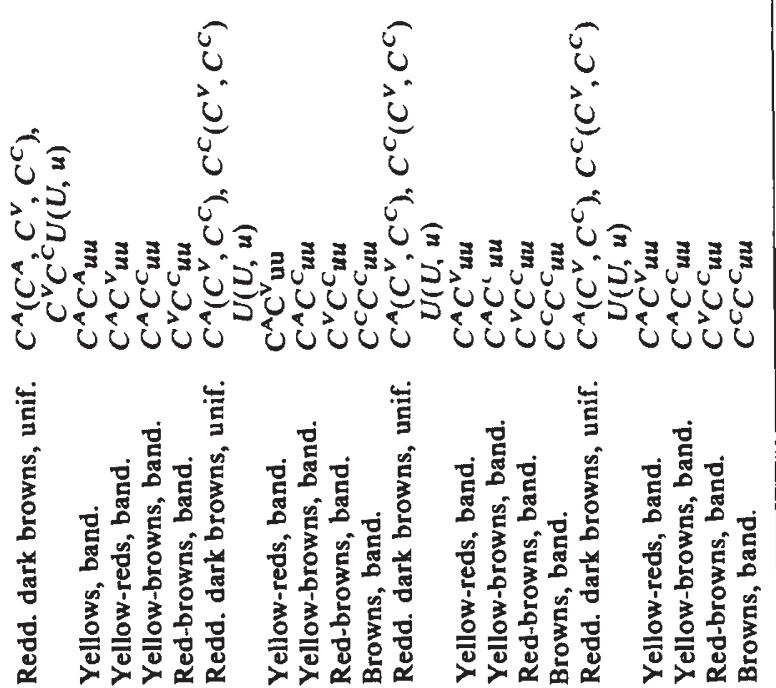

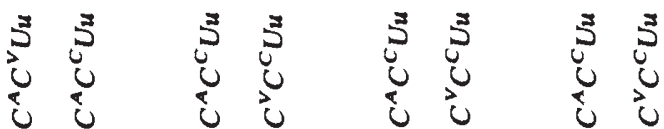

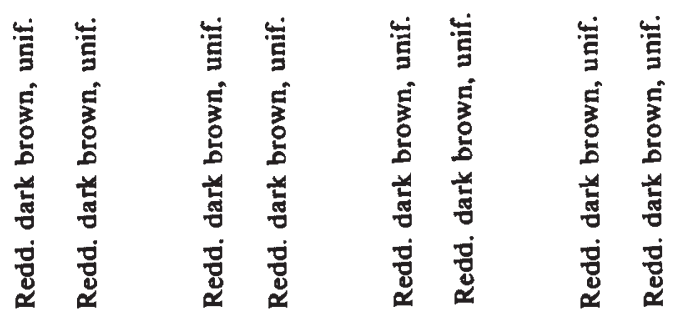

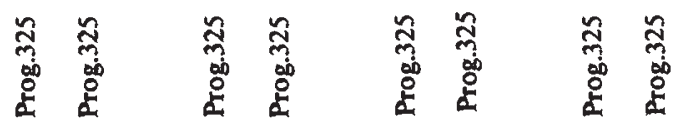

$\stackrel{\infty}{n} \quad \frac{0}{n} \quad \frac{\pi}{n}$ 
spots near the shell aperture where bands would end if they had developed. Also, effects on fascia albata, which is completely suppressed in $U U$ homozygotes, may be incompletely so in heterozygotes, which often show a short initial or juvenile fascia albata. $U U$ and $U u$ may also somewhat differ as to ground colour, $U u$ being less reddish than $U U$. However, as a rule the two classes largely overlap as to suppression effects on bands and darker upper part of the shell and also as to the reddish tinge, the results apparently depending on modifiers and the valency of the $U$ alleles in segregation. Especially in $U u$ heterozygotes, the shell after the protoconch may begin its development with the lighter colour (yellow, red, light brown) due to the $C$ alleles present in the genotype and then, at about $15-17 \mathrm{~mm}$ in width, the colour gradually shifts to the dark brown or the reddish dark brown determined by $U$.

\section{(b) Pleiotropic effects and a mutation from $u$ to $U$}

The threefold effects of $U$ appear not to be due to as many genes joined in a superlocus, but rather they must be pleiotropic. In the case of pleiotropy it may be expected that the locus includes special segments for each genic effect, but the separation of such segments depends on intragenic crossingover, much rarer than the crossing-over between the closely linked genes of a superlocus. In support of the interpretation of the effects of $U$ as being genuinely pleiotropic, we have recently detected in our cultures a mutation from $u$ to $U$ that changed the three effects at a time. Uniform had been completely absent from the strain where the mutant was observed.

Mated to a banded yellow-red sib (mating 471) the mutant proved to be heterozygous for $U$, as would be expected from an act of mutation that has occurred in a $u u$ genotype, and proved also to carry two alleles $C^{A}$ for yellow, as was shown by the observed progeny; reddish dark brown uniforms 51 , banded yellows 27 and banded yellow-reds 31 , bands being in all cases weakly pigmented because one $M^{0}$ should be present in the genotypes of the parents. It would be unlikely that two or three distinct genes, even of a superlocus would mutate concomitantly.

Acknowledgments. I am grateful to Professor J. A. Serra for help in the interpretation of results. Thanks are also due to Junta Nacional dos Produtos Pecuários, Lisbon, for support of the work made in the Centro de Genética e Biologia Molecular.

\section{REFERENCES}

AlbuQuerque De matos, R. M. 1979. Genética de alguns caracteres de Helix aspersa. Portug. Acta Biol., A, 15, 99-133.

ALBUQUERQUE DE MATOS, R. M. 1982. Interacção génica em caracteres pigmentares da concha de Helix aspersa. Portug. Acta Biol., A, 17, 37-85.

CAIN, A. J. 1971. Undescribed polymorphisms in two British snails. J. Conchol., 26, 410-416.

CLARKE, B., ARTHUR, W., HORSLEY, D. T. AND PARKIN, D. T. 1978. Genetic variation and natural selection in pulmonate molluscs. In Fretter, V. and Peake, J. (eds.) Pulmonates, vol. 2A, Academic Press, London, pp. 219-270.

COOK, L. M. 1969. Results of breeding experiments of Diver and Stelfox on Helix aspersa. Proc. malacol. Soc. Lond., 38, 351-358.

MURRAY, J. 1975. The genetics of Mollusca. In King, R. C. (ed.) Handbook of Genetics, vol. 3, Plenum Press, New York, pp. 3-31. 